\title{
Cognition in the African Grey parrot: Preliminary evidence for auditory/vocal comprehension of the class concept
}

\author{
IRENE M. PEPPERBERG \\ Purdue University, West Lafayette, Indiana
}

\begin{abstract}
An African Grey parrot, previously taught to discriminate more than 40 objects by means of vocal labels, has now acquired a limited understanding of abstract class concepts. The subject, Alex, can routinely decode and successfully reply vocally to queries concerning either color or shape for exemplars which simultaneously incorporate both variables (e.g.., colored and shaped keys, wood, and rawhide).
\end{abstract}

Recent studies (Pepperberg, 1981, Note 1, Note 2) have shown that training methods can be devised which enable the African Grey parrot to use its ability to reproduce the sounds of human speech in order to acquire a cognitive vocabulary consisting of vocal labels for several exemplars, actions, numerical quantities, instances of color and shape, and functional use of the word "no." While there are considerable anatomical, neurological, and behavioral differences between parrots and primates, our findings suggest that a particular avian species, the Grey parrot, can exhibit a number of mental and rudimentary communicative capabilities often thought to belong exclusively to humans and certain nonhuman primates (Fouts \& Couch, 1976; Gardner \& Gardner, 1978; Patterson, 1978; Premack, 1976; Savage-Rumbaugh, Rumbaugh, \& Boyson, 1980; Savage-Rumbaugh, Rumbaugh, Smith, \& Lawson, 1980); that is, our subject uses English vocalizations to identify proficiently, request, quantify, refuse, or comment upon more than 50 objects, and to alter, to a limited extent, his immediate environment (Pepperberg, 1981, Note 1, Note 2). Given that these findings on avian vocal behavior are contrary to the generally accepted view of "talking" birds as mere mimics (cf. Fromkin \& Rodman, 1974; Lenneberg, 1967), we are brought to the intriguing question of the extent of the mental capacity of the African Grey parrot with respect to cognition and communicatory ability. This report describes the results of a study designed specifically

This work was supported in part by NSF Grant BNS 7912945 and by the Harry Frank Guggenheim Foundation. I thank Kimberley Goodrich, Katherine Davidson, Denise Dickson, Mary Sandhage, Susan Reed, Gabrielle Cramer, Rebecca Cramer, and Bruce Rosen for their help as secondary trainers, and Nancy Mangini and two referees for their critical reviews of the manuscript. Portions of this paper were presented at the 1981 Animal Behavior Society meeting in Knoxville, Tennessee. The author's mailing address is: Department of Biological Sciences, Purdue University, West Lafayette, Indiana 47907. to investigate a particular (limited) aspect of the parrot's more abstract cognitive capabilities.

\section{Background}

Few researchers seem able to concur on the degree to which animals can exhibit an understanding of abstract cognitive concepts (cf. Griffin, 1981), probably because of the difficulties inherent in any attempt to establish an unequivocal hierarchy for relative levels of "abstraction." Nevertheless, many concede that, to have arrived at the so-called "higher levels," the animal subject in question must be able to respond not only to specific properties or patterns of stimuli (abstract though such individual instances may be)-e.g., just what is, or is not, "green" -but also to the classes or categories to which these specific properties belong, that is, to recognize the nature of the relationship that exists between a green pen and a blade of grass (Premack, 1978; SavageRumbaugh, Rumbaugh, Smith, \& Lawson, 1980; Thomas, 1980). We wished to learn if a nonprimate, nonmammalian organism could attain such relational understanding.

Our investigation necessarily began at one of the "lower levels" of abstraction (cf. Thomas, 1980) with the introduction of vocal markers for specific colors and shapes. Although individual instances of color and shape may be viewed as inherent physical (i.e., nonabstract) attributes of an object (e.g., "yellow" being the color of an optimally edible banana), experimental exemplars for which color and shape have no intrinsic relevance can be designed. Thus, a parrot would not be expected to receive differential intrinsic stimulation from two pieces of inedible rawhide, one blue and one green, but otherwise identical with respect to taste and surface feel; both would respond similarly when chewed or used for head scratching. Nor would there be any functional differences if one piece were square and the other triangular, as long as both had some number of corners; markers for such categorical prop- 
erties would then take on an abstract quality. By the end of the second year of our project, our avian subject, Alex, had learned, among other tasks, to discriminate several specific categorical instances. He was able to identify vocally (in response to "What's this?") 18 exemplars of diverse materials which varied with respect to any of three colors ["rose" (red), "green," "boo" (blue)] or two shapes ["3corner" (triangle) and "4-corner" (square)]; that is, he could appropriately label items such as green keys or triangular pieces of paper with an accuracy of $70 \%-80 \%$ (Pepperberg, 1981). He had also demonstrated a limited capacity to extend these discriminations to objects which differed somewhat from his training exemplars; for example, after training on blue key and blue hide, a newly dyed clothes pin was identified correctly as "blue peg wood," and, on first presentation, a large piece of computer output was identified correctly as "4-corner paper" (Pepperberg, 1981, Note 1). The question remained as to whether a parrot could proceed from recognition of particular instances of a category to recognition of the categorical concept itself. We chose to explore a relatively limited aspect of this problem: could our subject learn to associate labels representing the abstract categorical concepts ("color," "shape") with labels representing the various instances of these categories (e.g., "rose," "4-corner")? A means of examining this possibility (and thus further investigating the subject's understanding of the actual concepts of color and shape) would be to investigate his ability to recognize and respond differentially to queries of "What color?" and/or "What shape?" when presented with exemplars having instances of both color and shape (e.g., a green wooden triangle). We suggest that correct responses to different categorical queries concerning particular instances of color or shape for multivariant exemplars (ones which could equally well allow categorization with respect to either variable) would serve to elucidate some of the subject's more abstract conceptual abilities.

Note that the type of study proposed actually constituted a reclassification of objects: the same exemplar would have to be categorized with respect to shape in one instance and with respect to color in another. Reclassification is not only considered to be a more complex task than single-instance differentiation, but flexibility in changing the basis for classification is also thought to indicate the presence of "abstract aptitude"' (Hayes \& Nissen, 1956/1971).

\section{METHOD}

\section{Subject}

The experimental subject was an African Grey parrot that was obtained from a pet store in the Chicago area. The bird, named Alex, was approximately 13 months old at the start of our project in June 1977; prior to this date, he had received no formal vocal instruction. The parrot was allowed free access (contingent upon his vocal requests) to all parts of the laboratory for the $8 \mathrm{~h} /$ day that trainers were present; he was confined to a parrot cage $(\sim 62 \times 62 \times 73 \mathrm{~cm})$ during sleeping hours. Water and a standard seed mix for psittacines (sunflower seeds, dried corn, kibble, oats, etc.) were available continuously; fresh fruits, vegetables, specialty nuts (cashews, almonds, pecans, walnuts) and toys were provided at the bird's vocal request.

\section{Introduction of Relevant Labels}

Markers for various colors and shapes had been incorporated into our subject's vocabulary during the first and second years of our study, respectively, through specific training programs designed for that purpose (Pepperberg, 1981). Labels for the respective categorical classes were simultaneously introduced, but not as the subject of targeted instruction: such category labels were included intentionally, but in a casual manner, in vocal exchanges when appropriate. Thus, the parrot would have been exposed to vocal comments such as, "OK, Alex, let's work on colors today!", or, after he had been presented with a wooden (uncolored) triangle and had correctly responded to "What's this?", trainers might comment, "That's right, you know the shape; it's 3-corner wood." If the subject omitted the relevant marker when asked to identify a colored or shaped exemplar, examiners would ask, "What color X?", or "What shape Y?" As of the middle of the second year, one or two such queries were sufficient to elicit the appropriate response (Pepperberg, 1981).

However, such responses could not be taken as evidence that our parrot had acquired-either through such questions, for example, as in Premack's (1976) "no-fault tests," or as a serendipitous side effect of the relevant vocal exchanges described above-an understanding of the existence of any relation between such categorical queries and the targeted, missing markers: both types of queries could simply have been treated by the parrot as requests to "say something more." To explore explicitly the possible existence of any such associative learning (as well as the possible ramifications of such an ability in a nonprimate), we chose (1) to introduce, at various intervals during play sessions, items that were both colored and shaped, and to insert queries (either "What color?" or "What shape?") about such items into ordinarily scheduled reviews of his usual exemplars (in which all other questions would be posed as "What's this?"), and (2) to monitor, over the course of about 6 months, his responses. Such an approach would serve to integrate these tasks into his basic routine, and thus to mitigate what could have been an undue emphasis on presentations of these "multiplecategory" items-an emphasis which might have influenced, in some way, even these very preliminary findings.

We specifically decided against administering a rigorous singleinstance test of his comprehension at this stage (i.e., a test of his response to "What color?" and/or "What shape?" for a first-ever presentation of a colored and shaped item). Whether he had actually learned the connection between the label "color" and, for example, the label "rose" was not yet important, nor did we actually expect that such comprehension could yet exist. We were interested, at this point, only in the nature and extent of what he might have acquired, no matter how limited, and whether any understanding so acquired could be used as a starting point for further, targeted instruction.

Interestingly, Alex's responses to the very first instance of queries of "What color?" and "What shape?" for a newly crafted multivariant exemplar-a green wooden triangle-were correct ("green wood" and "3-corner wood," respectively); however, during subsequent (nontest) presentations of this object and similarly fashioned exemplars, he responded to the categorical queries appropriately, but somewhat less accurately, than during this initial exposure, that is, about $60 \%$. Given that he could choose his answers from two possible materials (wood and rawhide), three possible colors, and two possible shapes, and that we also considered as errors those responses for which the category was correct when the actual marker was wrong, his responses were above chance. His errors were not entirely inappropriate. Mis- 
takes were primarily separable into: (1) unclear vocalizations (undecipherable sounds, presumably for color or shape markers; e.g., "roween," a possible combination of "rose" and "green"), $4 \%$ of responses; (2) omissions of both markers (we termed these responses "generic"), $8 \%$ of responses; and, most frequently, (3) recitation of both appropriate color and shape markers, 12\% of responses. Other errors included: correct category but wrong specific marker, 4\%; incomplete shape marker (e.g., "corner wood" for 3-corner wood), 8\%; and wrong category, $4 \%$. Overall, the responses suggested that the parrot had perceived some relationship between the categorical concepts implied by the labels "color" and "shape" and their specific instances-for example, that "green" and "3-corner" represented different categories of markable attributes of a given exemplar-which we could use as a starting point for developing additional comprehension.

The observation that "type 3" errors were most prevalent suggested a particular focus for subsequent training. Our customary experimental protocol was such that, once training had begun on marked objects, Alex was not rewarded for unmarked responses if the exemplar was indeed colored or shaped. Our parrot would therefore have intentionally learned that only by providing all relevant data when asked to identify an object would he be considered correct. Consequently, failure of our subject to attend, and thus respond differentially, to our initial questions (i.e., "What color?"' or "What shape?", instead of the more familiar "What's this?') might have caused the prevalent "type 3" errors. While we could not as yet determine firmly if Alex had acquired an understanding of the abstract concepts, rather than of the particular instances of "color" and "shape," we could at least focus on developing and instituting training measures which would emphasize the content of the specific questions being posed. We would thereby (1) provide some additional training on the class category concept, (2) separate out possible training effects from conceptual knowledge, and (3) indirectly introduce the idea that targeted questions were also an integral part of communicatory behavior.

\section{Training Procedures}

Our specific training procedures and the rationale for their use have been described in detail elsewhere (Pepperberg, 1981). Our primary system, called the model/rival, or $M / R$, approach, is based on a protocol developed by Todt (1975), which in turn derives much from Bandura (1971) and Mowrer (1960). In this procedure, humans demonstrate to the parrot the types of interactive responses desired. Briefly, one human acts as a trainer of a second human, asking questions, giving praise and reward for correct answers, showing disapproval for incorrect answers (erroneous responses which mimic those being made by the bird at the time; e.g., "wood" for green wood); the second human acts both as a model for the bird's responses and as a rival for the trainer's attention. Roles of trainer and model are frequently reversed, and the parrot is given the opportunity to participate directly in these vocal exchanges. A typical interaction is reported in Table 1 .

A particularly crucial aspect of our work is that our procedures avoid use of extrinsic rewards (e.g., food rewards for correct identification of nonfood items); we focus instead on a selection of objects (toys) which themselves arouse the interest of the subject. Thus, each correct identification is rewarded by acquisition of the object itself, a system which permits the closest possible association of the object (or category) and the label to be learned.

\section{Criteria Prior to Testing}

Normally, our criterion as to when a newly acquired, targeted skill (e.g., the acquisition of a novel vocabulary item) can formally be tested is based on the clarity of the parrot's speech, and not on the accuracy of his performance of the task in question (Pepperberg, 1981). We are particularly careful to separate measurement of the efficacy of our training procedures in ex-

\section{Table 1}

Excerpt From M/R Training Session, Aprll 30, 1979

$\mathbf{K}:$

Kim, what color? (Holds up a green triangular piece of wood)

K: $\quad$ Green three-corner wood.

I: (Briefly removes object from sight, turns body slightly away). No! Listen! I just want to know color! (Faces back toward K; re-presents object) What color?

K: $\quad$ Green wood.

I: (Hands over exemplar) That's right, the color is green; green wood.

K: $\quad$ OK, Alex, now you tell me, what shape?

A: $\quad$ No.

K: $\quad$ OK, Irene, you tell me what shape.

I: Three-corner wood.

K: That's right, you listened! The shape is three-corner; it's three-corner wood (Hands over exemplar).

1: Alex, here's your chance. What color?

A: Wood.

I: That's right, wood; what color wood?

A: $\quad$ Green wood.

I: Good parrot! Here you go (Hands over exemplar). The color is green.

Note: I refers to the principal trainer, $K$ to Kimberley Goodrich, one of the secondary trainers, and $A$ to the parrot, Alex. This segment of the session lasted about 5 min.

panding our subject's vocal productivity from the success of such procedures in inculcating knowledge of the capacities we ultimately are to evaluate. In essence, we separate the effect our procedures have on our subject's ability physically to acquire a label from their effect on his ability to understand what the label represents. Only when the former skill is considered satisfactory do we deem the latter to be ready for testing (Pepperberg, 1981). This customary criterion could not be used in the current study, as the targeted responses (essentially the color and shape markers) were already produced clearly; our subject's understanding of their individual relevance had been independently tested for quite some time. A comparable criterion for deciding when to initiate formal testing on categorical concepts, however, could be to examine the efficacy of our modified training procedures in eliminating "type 3" errors (and, indirectly, "type 2"' as well; see Table 1), independently of an examination of the parrot's understanding of the relationship between the labels representing the abstract class concepts and the labels representing particular instances of such concepts. We therefore adopted the criterion that, in three successive sessions (outside of formal tests), the parrot respond with a single (but not necessarily categorically appropriate and/or specifically correct) marker $80 \%$ of the time when queried "What color?" or "What shape?" for exemplars exhibiting both features. Our subject could have responded (but did not) at chance levels to the queries "What color?" and "What shape?" and still have met this particular criterion had he employed only one marker in his response.

During this stage of the study, Alex appeared to lose interest during sessions composed entirely of color vs. shape queries, probably because the objects he would be acquiring were purposely designed so as not to offer much differential sensory stimulation; that is, although the questions revolved around items that exemplified various combinations of colors and shapes, there were only two "really" different objects for him to acquire: pieces of wood and pieces of rawhide. After just two or three queries, Alex would cease to work, begin to preen, or interrupt with many successive requests for other items (e.g., he would turn his back to the trainer, request corks, clothes pins, etc.). During the course of the study, we were also able to acquire colored, shaped keys, 
and such items were subsequently included on tests. However, colored and shaped pieces of paper could not be used as exemplars, as the parrot chewed on such items long enough to transfer the dyes (food colors applied by hand) to his beak, feet, feathers, perches, and ultimately to his trainers. Thus, after the criterion previously discussed was met, and testing could proceed, we examined his knowledge of class concepts by including, on each formal object-identification test, only one or two queries regarding color or shape for the various shape/color/object combinations. This procedure also acted as an additional precaution against "expectation cuing": in single-topic tests, contextual information (the homogeneous nature of questions that have a relatively restricted range of answers) could be responsible for a somewhat better performance than would otherwise be justified by a subject's actual knowledge of the topic.

\section{Test Procedures}

The details of our test protocol were presented in Pepperberg (1981). A brief outline is as follows: A secondary trainer, who is not involved in any instructional aspect of the project, presents to the bird, in a variable but previously determined random order, a fixed number of objects to be identified. The parrot is thus presented with an exemplar, asked "What's this?", "What color?", or "What shape?", and is then required to formulate a vocal English response. Trials are conducted by secondary trainers hired exclusively for that task to lessen the possibility of cuing (Pepperberg, 1981), and to keep the bird from applying its knowledge to maintain a bond with the principal trainer to the exclusion of other humans (Mowrer, 1952; Todt, 1975). The principal trainer is present, but sits in a corner of the room, does not look at the bird during the presentation of the test object, and therefore does not know what is being presented. In the rare case of accidental viewing of the object by the principal trainer, the secondary trainer substitutes another item from the list of exemplars to be tested that day, that is, reorders the exemplars. The principal trainer repeats what she heard the bird say; if that is, indeed, the name of the presented object, the parrot is rewarded with praise and by being allowed to inter- act with the object for as long as desired (usually about $1.5 \mathrm{~min}$ ). There are then no additional presentations of the object during the test. If the identification is incorrect (or indistinct), the examiner removes the object, turns his/her head, and emphatically says "No!" The misnamed object is then immediately repeatedly presented until a correct identification is made; any errors are recorded. Thus, the parrot finds (and appears to learn) that an incorrect identification, for example, substitution of the name of a more desired object for the one presented, is fruitless; instead, a quick, correct identification allows him to proceed to a preferred item. We determine the "desirability" of particular objects by the number of times these items are requested during the free periods (nontaping, nontesting, nontraining sessions) and by the length of time these items are handled and chewed after they have been acquired by the parrot. Because immediate repeated presentation of an object during a test occurs only when the response to the initial presentation is incorrect, the testing protocol penalizes the use of a win-stay, lose-shift strategy. Repetition of a previously correct response, for example, the name of the previous exemplar, elicits no reward. Our testing procedure thus provides a definite contrast to our training protocols, which rely on, and occasionally reinforce, repetitive behaviors. At all stages, the overall test score is obtained by dividing the number of ultimately correct identifications (i.e., the predetermined number of objects in the test) by the total number of presentations required.

\section{RESULTS AND DISCUSSION}

More than 150 formal tests included queries of "What color?" and/or "What shape?" for exemplars which represented various combinations of three different colors, two different shapes, and three different materials (wood, rawhide, and metal key tops). Results are presented in Table 2. Alex's scores for such identifications (e.g., queries that required answers of "rose wood" or "3-corner hide") were

Table 2

Test Results for "What Color?" vs. "What Shape?" Comprehension

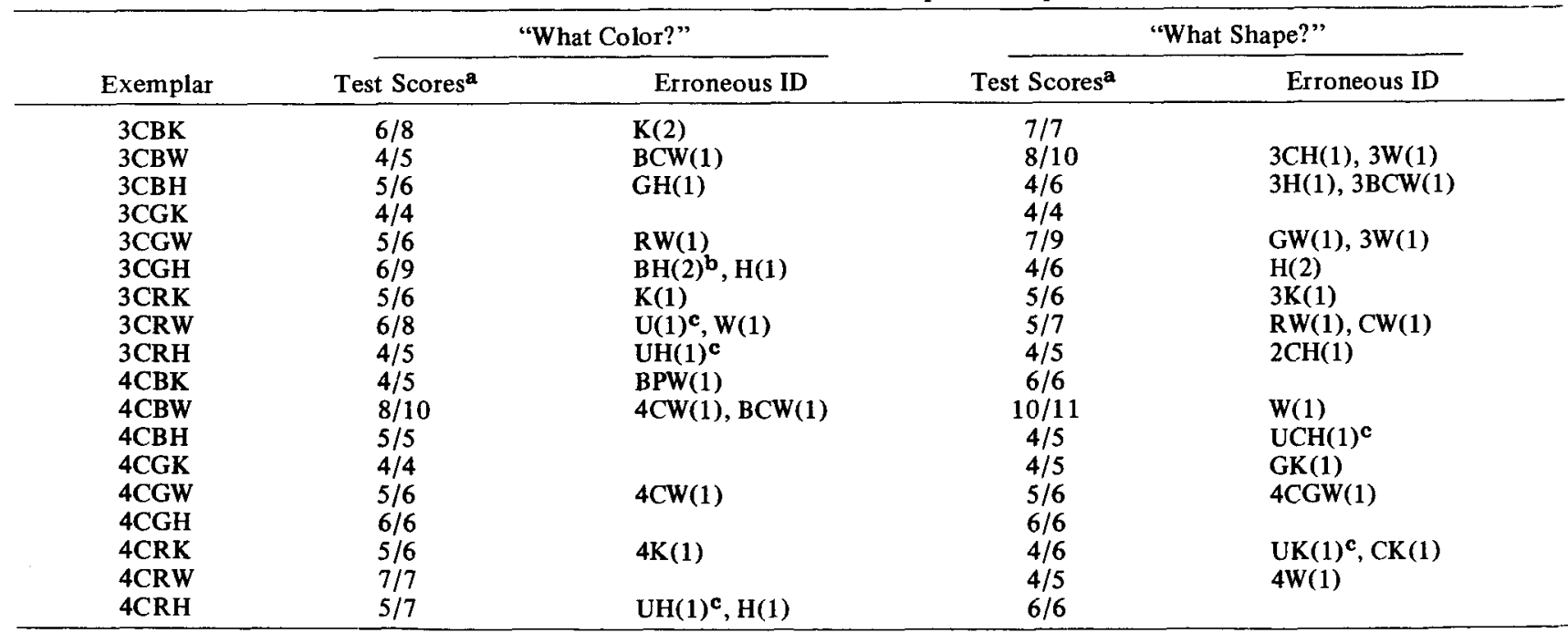

Note-Each identification test averages one such question for a colored and shaped object (e.g., Alex is shown a rose three-cornered piece of wood and asked either "What color?" or "What shape?"). These results are from 161 different tests: $9 / 10 / 79-9 / 1 / 82$.

Code $: C=$ corner; $R=$ rose $; G=$ green $; B=$ blue $; W=$ wood $H=$ hide $;=k e y ; P W=$ peg wood. ${ }^{a}$ Test scores are number of correct identifications divided by the total number of presentations. Overall, Alex achieved scores of $84.7 \%$ (colors) and $83.7 \%$ (shapes). ${ }^{b}$ We noted that one of the exemplars was bluish in tone; after replacing it, these errors ceased. ${ }^{c}$ The parrot's production of the marker was unintelligible. Erroneous ID = erroneous identification (number of times error made). 
Table 3

Test Results for "What Color?" vs. "What Shape?" Comprehension for Exemplars Which Vary With Respect to Recently Introduced Colors and/or Shapes

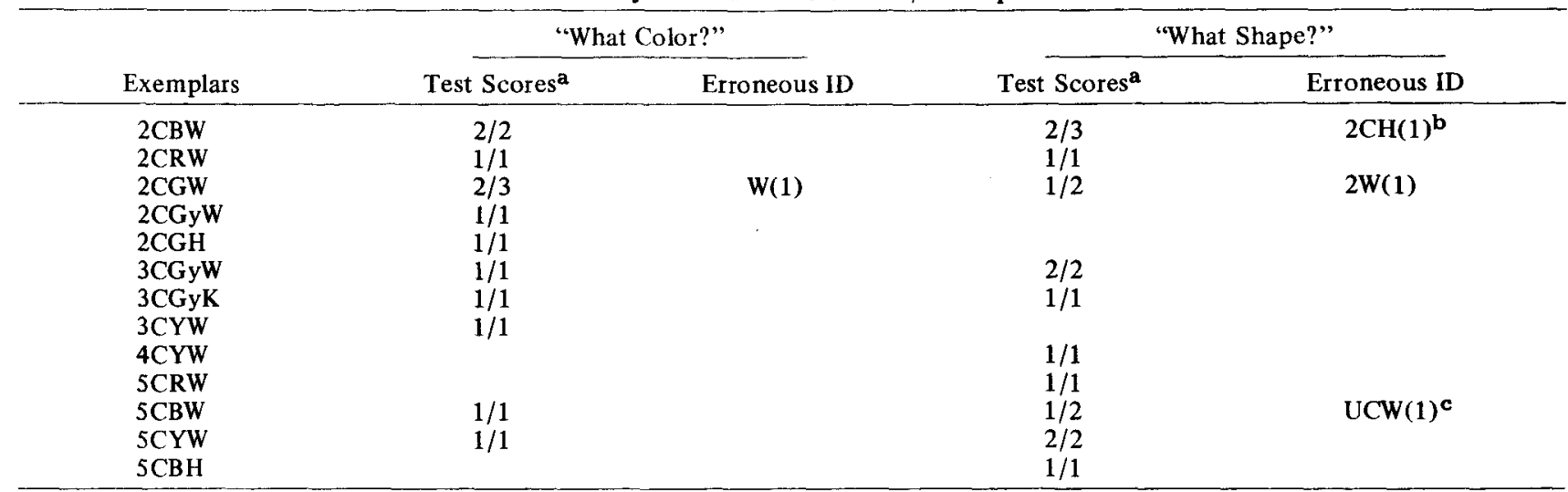

Code: $C=$ corner $R=$ rose $G=$ green $; B=$ blue $; G y=$ gray $; Y=$ yellow $; W=$ wood $; H=$ hide $; K=$ key. ${ }^{a}$ Test scores are number of correct identifications divided by the total number of presentations. ${ }^{b}$ Error was made on initial presentation. ${ }^{c}$ The parrot's production of the marker was unintelligible. Erroneous $I D=$ erroneous identification (number of times error made).

better than $80 \%$. We have recently added test items that allow variation in two additional colors (gray and yellow) and/or two additional shapes [2-corner (football) and 5-corner (pentagon)]. Those results are presented separately in Table 3.

The results demonstrate that our subject can view an item which can be described relevantly in more than one way, decode from our question which of two categories is being targeted, and then produce, based on the question posed, the appropriate instance of that category as an answer. Moreover, a change in the target of our question causes him to reclassify the exemplar, either immediately or during presentations separated by several days.

We wish to point out that the immediate focus (and therefore the test protocol) of our study was quite different from, for example, the investigation of symbolic categorization reported in SavageRumbaugh, Rumbaugh, Smith, and Lawson (1980), although the fundamental objectives of both projects were the same, viz, to determine if our respective nonhuman subjects could associate symbolic categorical labels and their symbolically represented instances. We chose a different means of exploring this ability. Our particular objective was not to determine if our subject, after learning to reference symbols for instances of a category, $a_{i}$, with the symbol for the category itself, A (likewise, $b_{i}$ and B), could properly symbolically categorize novel $\mathrm{a}_{\mathrm{i}} \mathrm{s}$ and $b_{i} s$. We wished, instead, to determine if our subject could decode symbolic representations (vocal questions) of two categories of abstract attributes (color, shape) when both categories were simultaneously relevant. That is, our emphasis was not on encoding a sorting task symbolically, but on decoding appropriate symbolic questions. This distinction is particularly important when evaluating our test protocol, that is, our presentation of the same exemplars on multiple (and often successive) tests. For our purposes, a "transfer test" to novel stimuli (i.e., whether Alex would respond "yellow wood" to "What color?" for a novel yellow pentagon after learning the labels "yellow" and "5-corner") would not be particularly informative. Rather, we had to determine if he could reliably decode, each time, our queries about each exemplar. Reward for correct classification of green triangular wood as "green wood" in one instance and as "3-corner wood" on another would provide little information as to how to respond to a third query. However, we do note that, for first-time-ever presentations of objects that varied in newly acquired colors and/or shapes, Alex scored $12 / 15$ on queries of either "What color?" or "What shape?" for the newly acquired attributes. His errors were, for the most part, not directly related to the categorical concepts under consideration; they are listed in Table 3.

While our findings suggest that Alex has acquired a limited understanding of the concepts of color and shape, we must stress the rudimentary nature of his accomplishments. Although he has recently incorporated the actual labels "color" and "shape" into his repertoire, he is just beginning to respond appropriately to such queries as "How do $X$ and $Y$ differ?"', and we have not yet presented him with pictures of collections of colored and shaped exemplars (e.g., a green wooden pentagon, a rose rawhide triangle, and a blue key with a square face) and asked "What's rose?" or "What shape is key?" (cf. Essock, Gill, \& Rumbaugh, 1977). Also, given his still limited vocabulary size and comprehension, we cannot yet present such questions as "Is an apple yellow?" when no fruit is available and expect him to answer appropriately. And, because productive use 
of a learned concept is another measure of cognitive ability, we note that there has been only a single instance in which Alex has used the phrase "What color?" as a learning tool: He queried a student as to the color of his mirror image. After hearing replies such as "That's grey; you're a Grey parrot" in response to six such inquiries, he acquired the label "grey" and subsequently used it to identify correctly, on first showings, newly dyed grey exemplars. ${ }^{1}$

Also, although we stress that there exist distinctions between the forced-choice conditional discrimination tests often used to investigate nonhuman conceptual abilities and our test protocol, which involves referential (vocal) labeling (cf. Pepperberg, 1981, Note 1), we note that Alex may have learned a form of conditional response: For example, "If I hear ' $A$ ' (a categorical label), I must respond with an ' $a$ ' (a label for an instance of that category)." But this performance implies, we claim, that our subject had acquired at least a rudimentary concept of the relationship between the category $A$ and its instances, the " $a_{1}$ " as opposed to $B$ and the " $b_{1}$." Moreover, comprehension and utilization of such a vocal conditional constraint on the already inherently conditional nature of the productive labeling task (cf. Premack, 1976, pp. 140-142) suggest that our subject's accomplishments represent a significant (cognitive) achievement.

We believe that the present study has demonstrated that an African Grey parrot can form a relationship (albeit rudimentary) between the categorical concepts implied by the labels "color" and "shape" and their specific instances, that is, that a specific color and a specific shape represent different categories of markable attributes of an individual item. This skill is not just the ability to recognize a particular instance of a category (e.g., "greenness"), or even to perform a conditional discrimination (e.g., to match shapes under one stimulus and colors under another), but rather the ability to extract the relevant category from a multivariant item and respond with the label for the one, correct instance of this category. Acquisition of categorical concepts, rather than categorical instances, is viewed by many researchers to imply advanced cognitive capabilities (Premack, 1978; Thomas, 1980). While we are not attempting to find where our subject may fit on any relative "intelligence" scale, we do believe that consideration of the hierarchies by which researchers attempt to assess intelligent behaviors enables the accomplishments of our subject to be better understood.

\section{REFERENCE NOTES}

1. Pepperberg, I. M. A study in interspecies communication: Evidence for cognitive and verbal abilities in the African Grey parrot. In H. Markowitz (Chair), Comparative psychology: Communication. Symposium presented at the meeting of the Western Psychological Association, Sacramento, April 1982.

2. Pepperberg, I. M. Evidence for numerical counting ability in the African Grey parrot (Psittacus erithacus). Paper presented at the annual meeting of the Animal Behavior Society, Ft. Collins, Colorado, June 1980.

\section{REFERENCES}

Bandura, A. Analysis of modeling procedures. In A. Bandura (Ed.), Psychological modeling. Chicago: Aldine-Atherton, 1971.

Essock, S. M., Gill, T. V., \& Rumbaugh, D. M. Language relevant object- and color-naming tasks. In D. M. Rumbaugh (Ed.), Language learning by a chimpanzee. New York: Academic Press, 1977.

Fours, R. S., \& Couch, J. B. Cultural evolution of learned language in chimpanzees. In M. E. Hahn \& E. C. Simmel (Eds.), Communicative behavior and evolution. New York: Academic Press, 1976.

Fromkin, V., \& Rodman, R. An introduction to language. New York: Holt, Rinehart and Winston, 1974.

Gardner, R. A., \& Gardner, B. T. Comparative psychology and language acquisition. In K. Salzinger \& F. L. Denmark (Eds.), Psychology, the state of the art. New York: Annals of the New York Academy of Sciences, 1978, 309, 37-76.

Grirfin, D. R. The question of animal awareness. New York: Rockefeller University Press, 1981.

Hayes, K. J., \& Nissen, C. H. Higher mental functions of a home-raised chimpanzee. In A. Schrier \& F. Stollnitz (Eds.), Behavior of nonhuman primates (Vol. 4). New York: Academic Press, 1971.

LENNEBERG, E. Biological foundations of language. New York: Wiley, 1967.

Mowrer, $\mathbf{O}$. H. The autism theory of speech development and some clinical applications. Journal of Speech and Hearing Disorders, 1952, 17, 263-268.

Mowren, O. H. Learning theory and symbolic processes. New York: Wiley, 1960.

Patpenson, F. Linguistic capabilities of a lowland gorilla. In F. C. C. Peng (Ed.), Sign language acquisition in man and ape (AAAS Symposium). Boulder, Colo: Westview Press, 1978.

Peppenberg, I. M. Functional vocalizations by an African Orey parrot (Psittacus erithacus). Zeitschrift für Tierpsychologie, 1981, 55, 139-160.

Premack, D. Intelligence in ape and man. Hillsdale, N.J: Erlbaum, 1976.

Premack, D. On the abstractness of human concepts: Why it would be difficult to talk to a pigeon. In $\mathbf{S}$. $\mathbf{H}$. Hulse, $H$. Fowler, \& W. K. Honig (Eds.), Cognitive processes in animal behavior. Hillsdale, N.J: Erlbaum, 1978.

Savage-Rumbaugh, E. S., Rumbaugh, D. M., \& Boysen, S. Do apes use language? American Scientist, 1980, 68, 49-61.

Savage-Rumbaugh, E. S., Rumbaugh, D. M., Smith, S. T., \& LAwson, J. Reference: The linguistic essential. Science, $1980,210,922-925$.

TномАв, R. K. Evolution of intelligence: An approach to its assessment. Brain, Behavior and Evolution, 1980, 17, 454-472.

ToDT, D. Social learning of vocal patterns and models of their applications in Grey parrots. Zeitschrift für Tierpsychologie, 1975, 39, 178-188.

\section{NOTE}

1. We suspect that such prompt learning was facilitated by his prior acquisition of the requisite phonemes: "gr" from "green" and " $\overline{\mathrm{a}}$ " from "pāper"; vocalizations that involve instead novel phoneme production may require 1-2 months of training before 
becoming entirely clear. We do not mean to suggest that our subject actually recognizes and recombines human phonemes. We do mean that, in order to acquire labels that include new sets of sounds-most easily described as phonemes-our subject seems to need to develop new forms of motor control for syrinx, tongue, and beak. Once this control has been acquired, it can be transferred to other labels.

(Manuscript received June 18, 1982; revision accepted for publication December 13, 1982.) 